\title{
Pain Neuroscience Education in cancer survivors with persistent pain: A pilot study
}

\author{
Roselien Pas ${ }^{\mathrm{a}, \mathrm{b}, \mathrm{f}}, \mathrm{PT}, \mathrm{MSc}$, Laurence Leysen ${ }^{\mathrm{a}, \mathrm{b}}$, PT, MSc, Wanda De Goeij ${ }^{\mathrm{be}}$, \\ PT, Leonieke Vossebeld ${ }^{\mathrm{be}}$, PT, Paul Van Wilgen ${ }^{\mathrm{a}, \mathrm{d}}$, PT, PhD, An De Groef ${ }^{\mathrm{g}}$, PT, \\ $\mathrm{PhD}$, Margot De Kooning ${ }^{\mathrm{a}, \mathrm{b}, \mathrm{c}}$, PT, PhD
}

${ }^{a}$ Pain in Motion International Research Group, www.paininmotion.be;

${ }^{b}$ Department of Physiotherapy, Human Physiology and Anatomy (KIMA), Faculty of Physical Education \& Physiotherapy, Vrije Universiteit Brussel, Brussel, Belgium;

${ }^{c}$ Department of Physical Medicine and Physiotherapy, University Hospital Brussels, Brussel, Belgium;

${ }^{d}$ Transcare Transdisciplinary Pain management Centre, Groningen, the Netherlands

${ }^{e}$ The Berekuyl Academy, Harderwijk, the Netherlands

${ }^{f}$ Department of Rehabilitation Sciences and Physiotherapy, Faculty of Medicine and Health Sciences, University of Antwerp, Antwerp, Belgium

${ }^{g}$ KU Leuven - University of Leuven, Department of Rehabilitation Sciences and University Hospitals Leuven, Department of Physical Medicine and Rehabilitation, Leuven, Belgium

Address correspondence to: Roselien Pas, Vrije Universiteit Brussel (VUB), Department of Physiotherapy, Laarbeeklaan 103, 1090 Jette, Belgium, Tel:02/477.45.29, Fax:02/6292876 roselien.pas@vub.be

Declaration of interest statement: The authors have no conflicts of interest to declare. 


\title{
Pain Neuroscience Education in cancer survivors with persistent pain: A pilot study
}

\begin{abstract}
Purpose: To describe the Pilot Study: Pain Neuroscience Education in Cancer Survivors and describe the innovative educational component of Pain Neuroscience Education (PNE).

Design: Quasi experimental design

Method: The PNE program, encompassing a one-on-one education session and an information leaflet was given to 30 cancer survivors. At baseline and two weeks after the PNE, participants were asked to fill out following outcome measures; pain intensity, pain catastrophizing, and HRQoL.

Findings: Following PNE, a significant decrease on pain intensity $(p=0.001)$, on the SF-36 subscale pain $(p=0.003)$ and for the following PCS subscales: Helplessness $(p<0.001)$, Rumination $(p=0.002)$ and Total score $(p<0.001)$ was found compared to baseline.

Conclusions: Although the current results need to be verified in a larger randomized, controlled trial, preliminary evidence shows a decrease in pain intensity and pain catastrophizing following PNE in cancer survivors with persistent pain.
\end{abstract}

Keywords: cancer survivors; chronic pain; pain education; pain catastrophizing; pain intensity; quality of life 


\section{INTRODUCTION}

Cancer is a health problem worldwide. The Union for International Cancer Control (GLOBOCAN) estimates 18.1 million people were diagnosed with cancer in 2018 and this incidence rate is expected to increase to 21.4 million new cancer patients by 2030 (Ferlay et al 2018; Ferlay et al 2010). Fortunately, more people survive cancer due to new developments in cancer diagnosis and treatment (Gatta et al 2014). Despite the probability of surviving cancer, many cancer survivors are confronted with unfavorable disease- and treatment-related sideeffects; which can result in prolonged pain (Cleeland et al 2012; Glare et al 2014; Harrington, et al 2010). For example, some breast cancer treatment-related effects are; adhesive capsulitis, cervical radiculopathy, radiation fibrosis and lymphedema (Cleeland et al 2012).

Approximately $36 \%$ of breast cancer survivors experience pain symptoms following his/her cancer treatment, which may last for several months, years, or even a lifetime (Leysen et al 2018). Persistent (i.e. chronic) pain, defined as the presence of pain for at least 3 months (Merskey \& Bogduk, 1994), influences the cancer survivors' Health-Related Quality of Life (HRQoL) and exposes them to an increased risk for depression and anxiety (Bredal et al 2014). Furthermore, a considerable number of cancer patients indicate that they do not have enough knowledge regarding pain during or after cancer treatments, what the possibilities of pain relief are; and how to access help when needed (Binkley et al 2012; McGuire 2004). Cancer survivors suffering from persistent pain, should be treated as fast as possible and in the most optimal way. Currently, pain management in cancer survivors includes a multidisciplinary approach consisting of an individually tailored combination of medication, physical therapy and psychosocial interventions (Glare et al 2014; Levy et al 2008; Syrjala et al 2014). In most cases, patient education is embedded within one of the aforementioned interventions (Lovell et al 2014). Evidence exists that pain education improves the knowledge of pain and reduces pain 
intensity in cancer patients (Adam et al 2015). However, these educational interventions are directed towards cancer patients suffering from pain during the treatment phase rather than the post-treatment phase (Bennett et al 2009). Consequently, the content of these educational interventions is restricted to more biomedical pain management instructions, including tips on analgesic usage, enhancing pain assessment and tackling barriers to analgesic non-adherence (Bennett et al 2009). Therefore, the educational content of these programs might be less relevant for cancer survivors with persistent pain.

During and after their cancer treatment, the cancer patient is faced with many challenges: (a) a new body image; (b) self-image; and (c) waiting on additional diagnostic findings (Hungr et al 2017). The additional burden of persistent pain is an extra stressor to cope with and makes it more difficult for the patient to resume their daily life activities (Kudel et al 2007; Sun et al 2017). Therefore, breast cancer survivors might benefit from a more biopsychosocial explanation of pain (Malfliet et al 2017). The misunderstanding of pain holds several consequences for the cancer survivor itself; they consider pain to be more threatening, they present with lower pain tolerance, and they have more catastrophic thoughts and less adaptive coping strategies which might result in worse treatment outcomes (Malfliet et al 2017). The goal of the innovative project, Pain Neuroscience Education, is to provide information regarding the biological and physiological aspects of pain, and to discuss myths and negative views of pain. During the one-on-one session, various neurophysiological pain concepts are explained to the patient by using pictures and metaphors. More specifically, the PNE session provided information about the central and peripheral nervous system; the changes that occur in the case of chronic pain; and the influence of stress and emotions on pain perception (Nijs et al 2018). 
Firm evidence exists for the effectiveness of PNE in decreasing pain intensity, pain catastrophizing and increasing HRQoL in several non-cancer populations with chronic pain, such as musculoskeletal pain, osteoarthritis and chronic whiplash associated disorders (Louw et al 2016; Meeus et al 2010; Van Oosterwijck et al 2011). A systematic review investigating different kinds of pain educational interventions in adults with chronic pain, concluded that a PNE approach significantly improves pain catastrophizing and knowledge of pain (Geneen et al 2015). Thus, the content of the pain education interventions is of great importance, with most benefits when the content is adjusted to the specific pain population (chronic neck -and low back pain, post-cancer pain,...)(Louw et al 2016; Malfliet et al 2017).

To date, it is still unknown whether PNE is beneficial in cancer survivors with persistent pain after finishing active cancer treatment. The purpose of this paper is to describe the Pilot Study: Pain Neuroscience Education in Cancer Survivors and describe the innovative educational component of Pain Neuroscience Education (PNE). The innovative educational component/project consists of two components, a one-on-one education session and an educational leaflet.

\section{METHODS}

\section{Cancer survivors}

Cancer survivors who met following eligibility criteria were selected; (1) 18 years or older; (2) self-reported chronic pain, defined as the experience of pain for a period of at least 3 months; (3) last session of their primary cancer treatment (surgery, radiotherapy, chemotherapy and immunotherapy) at least three months ago. In addition, the cancer survivors had to be able to understand, read, and speak the Dutch language. Cancer survivors who reported pain before cancer diagnosis and/or previously followed a pain educational program were excluded. According to the National Cancer Coalition for Survivorship (NCCS), "an individual is 
considered a cancer survivor from the moment of diagnosis and for the balance of his or her life, regardless of the ultimate cause of death" (1986). Throughout this paper, however, 'cancer survivor' refers to an individual with a history of cancer who is beyond the acute diagnosis and treatment phase.

\section{Procedure}

Figure 1 provides an overview of the timetable of the study protocol. This study was conducted in compliance with Dutch law and the principles of the Declaration of Helsinki. Therefore, no specific ethical clearance was needed. Recruitment took place in five private physiotherapy practices in the Netherlands (Roermond, Maasbracht, Winterswijk, Enter, and Boekelo) between November the $1^{\text {st }}$ (2017) until the end of January (2018). Cancer survivors, who met the eligibility, criteria were invited to participate and subsequently asked to sign the informed consent after receiving the necessary information about the study. The cancer survivors were discouraged to start new treatments on top of their current ongoing treatments (medication, physiotherapy) during the two-weeks duration of the study. At the first meeting, demographic data were collected by using a questionnaire. In addition, they were asked to complete questionnaires on pain intensity, pain catastrophizing, and HRQoL. During the same meeting, the cancer survivor received a one-on-one education session. At follow-up, two weeks later, the cancer survivors were asked to complete the pain intensity, pain catastrophizing, and HRQoL questionnaires again. Outcomes were assessed by the two same therapists who were giving the 


\section{Measurements and Questionnaires}

Pain intensity, pain catastrophizing, and HRQoL were recorded by the Visual Analogue Scale (VAS), Pain Catastrophizing Scale (PCS), and the Short Form-36 Health Status Survey (SF36), respectively.

The VAS consists of a 100 millimeter $(\mathrm{mm})$ long line and the score ranges from 0 ; no pain at all to 100 ; the most unbearable pain. The cancer survivors were instructed to rate the average pain intensity during the last week. The VAS shows good associations with other pain intensity ratings (criterion validity $r>0.70$ ) and is a reliable (test-retest coefficient $=0.80$ ) method to score pain intensity in cancer patients (Jensen 2003).

The PCS is a 13-item questionnaire, measuring catastrophic thinking about pain. The thirteen items are scored on a five-point scale, with a total score being calculated by combining the scores on each individual item. Higher scores correspond to more severe catastrophic thoughts and feelings about pain. In addition, the total score can be subdivided into three subscales: rumination, magnification, and helplessness. The PCS is found to be internally consistent (Cronbach's alpha; Total score $=0.87$, rumination $=0.84, \quad$ magnification $=0.72$, helplessness $=0.89$ ) and is reliable over time (test-retest coefficient $=0.70)$. Its validity is wellestablished with an invariant factor structure across pain samples (Van Damme et al 2002). The PCS has been used in several cancer pain studies to measure pain catastrophizing (De Groef et al 2018; Lee et al 2017).

The SF-36 was used to measure the cancer survivors' HRQoL. This questionnaire consists of 36 questions, which can be divided into nine subscales that represent nine domains of health (Physical Functioning, Social Functioning, Role limitations due to Physical health, Role 
limitations due to Emotional health, Emotional wellbeing, Energy \& Fatigue, Pain, General Health Perception, and Health Changes). The cancer survivors answer each question using an ordinal scale (0-3 or $0-6$, depending on the question). These numerical answers are then recoded to yield scores ranging from 0 to 100 , indicating improvement as the scores increase. For each of the nine domains, a score can be generated. The SF-36 is a reliable (Cronbach's alpha $>0.70$ ) and valid instrument among breast cancer survivors (Treanor \& Donnelly, 2014).

\section{Intervention}

The intervention consisted of one (one-to-one) educational session, lasting for 30-45 minutes. The intervention was delivered by two therapists with a bachelor's degree in physiotherapy, who received specific courses in pain neurophysiology and who had at least 7 years of clinical physiotherapy experience. Both therapists were guided by a therapist with a master degree in physiotherapy and expertise in PNE. The content of the PNE intervention was taught by using a PowerPoint presentation on a computer. An example the PNE supporting material can be found on the PainInMotion website http://www.paininmotion.be). The computer presentation provided information pertaining to the physiology of the nervous system and pain. In addition, the following topics were included in the educational session; characteristics of acute versus chronic pain; the purpose of acute pain; how acute pain originates in the nervous system; how pain becomes chronic; and potential sustaining factors of persistent pain like emotions, stress, pain cognitions, and pain behavior. Cancer survivors also received an information leaflet about the neurophysiology of pain, containing the same information as discussed during the PNE session and were encouraged to reread the information at home after the educational session. 


\section{$\underline{\text { Statistical analysis }}$}

Statistical analyses were performed using Statistical Package for Social Sciences (SPSS) 25.0 (SPSS Inc., Chicago, IL USA). Appropriate descriptive statistics were calculated (mean \pm SD). The normality of data was examined using the one-sample Kolmogorov-Smirnov test. Since variables were not-normally distributed, hence non-parametric analysis were used. In order to account for missing data, all analyses were performed using the 'last observation carried forward' method for intention-to-treat analysis. Pre-treatment data and post-treatment data of the VAS, PCS, and SF-36 were analyzed using the Wilcoxon signed-rank test to examine the treatment result of the PNE. The significance level was set at 0.05 . The effect size (ES) $r$ was calculated by the formula of Rosenthal $r=\mathrm{Z} / \sqrt{ } \mathrm{N}$ because data distribution violated the general assumptions of Cohen's formula (Rosenthal 1994). The r-value, represents a correlational effect and has different interpretation thresholds compared to its parametric counterpart Cohens-d. Hence interpretation intervals for the r-value are $0.10-0.29$ small, $0.30-0.49$ medium, $0.50-0.69$ large and above 0.70 as a very large effect size (Rosenthal 1996). Since the r-value is a correlation effect, effect sizes can be both positive and negative.

\section{$\underline{\text { RESULTS }}$}

\section{Cancer survivors}

A total of 30 cancer survivors participated in the study with a mean age of 62.97 years $( \pm 13.62$ SD). Twenty-four women $(n=24)$ and six men $(n=6)$ were included. Seven different cancer types were included, with breast cancer being the most prevalent cancer type $(n=19 ; 63 \%)$. Other cancer types were prostate cancer $(n=3)$, colorectal cancer $(n=2)$, brain tumor $(n=2)$, stomach cancer $(n=2)$, cervical cancer $(n=1)$, and neck cancer $(n=1)$. Twenty-three women $(n=23)$ and six men $(n=6)$ completed the pilot study. One participant, due to personal reasons, did not complete the study. 


\section{Effect of PNE on Pain Intensity}

Levels of the VAS were significantly higher pre-PNE compared to post-PNE $(p=0.001, r=-$ 0.44). Correspondingly, the Pain subscale from the SF-36 was significantly lower before PNE $(p=0.003, r=-0.39)$. Detailed results can be found in table 1 .

\section{Effect of PNE on Pain Catastrophizing}

The total score of the PCS was significantly lower after PNE $(p<0.001, r=-0,46$.). In addition, two of the three subscales of the PCS significantly decreased after PNE, being the rumination subscale $(p=0.002, r=-0.39)$ and the helplessness subscale $(p<0.001, r=-0.48)$. However, the magnification subscale did not differ significantly from baseline $(p=0.095, r=-0.22)$ (details in table 1).

\section{Effect of PNE on Health-related Quality of Life}

The nine subscales were analyzed separately to evaluate the effect of PNE on HRQoL. Excluding the subscale pain, none of the additional eight subscales demonstrated significant changes after PNE. Detailed results can be found in table 1.

\section{$\underline{\text { DISCUSSION }}$}

To our knowledge, this is the first study exploring the use of a PNE program in cancer survivors with persistent pain. After one PNE session, positive effects were found on pain catastrophizing and pain intensity. 
The benefit of PNE for cancer survivors with persistent pain is similar with previous findings in research with non-cancer chronic pain populations (Louw et al 2016; Meeus et al 2010). Analogous to the non-cancer chronic pain population, pain catastrophizing in cancer patients is associated with an intensified experience of acute pain during cancer treatment, and greater likelihood of significant persistent pain after cancer treatment (Schreiber et al 2014).

Results of this study showed medium to large effects of PNE on the total PCS score and its subscales 'rumination' and 'helplessness'. These findings are in line with previous studies examining PNE in non-cancer chronic pain populations (Louw et al 2016; Meeus et al 2010). Five studies, investigating PNE as an education-only intervention in chronic musculoskeletal pain, failed to find a positive effect of PNE on pain ratings (Louw et al 2016). In contrast, this pilot study showed a significant reduction in pain intensity, as well as positive effects on the subscale 'pain' of the SF-36. Further research should investigate whether these discrepant findings are due to any differences in treatment protocol or the heterogeneous population sample used in this study. For example, patients with chronic musculoskeletal pain mostly present with a dominant central sensitization type of pain (Clauw 2015), while cancer survivors with persistent pain typically suffer from neuropathic type or mixed pain type (i.e. nociceptive, neuropathic pain and/or central sensitization pain) (Leysen et al 2018). The presence of different types of pain experienced within the study population, resulting from different treatment-related morbidities, such as lymphedema, radiation fibrosis and adhesive capsulitis, might have influenced the results in this pilot study.

This study has some limitations. Practical guidelines for the implementation of PNE in cancer patients with persistent pain suggest that pain education should include at least two education sessions to give the opportunity to discuss the information leaflet (Nijs et al 2018). One 
limitation of this study was the number of sessions. This pilot study consisted of only one educational session, which prevented the patients the opportunity to ask questions during the days after the intervention. The preliminary results of this pilot should be interpreted with caution and should not be generalized to the total cancer survivor population given the use of its small and convenience sample size.

Although the patients were encouraged not to start new therapy modalities for their chronic pain complaint, this was not addressed by a self-reported question of item. Secondly, because of ethical considerations, it was not possible to deprive patients from ongoing physiotherapy treatments, resulting in $67 \%$ percent of the patients continuing treatment (manual lymphatic drainage or exercise therapy) while completing the study protocol. The promising preliminary results of this pilot study should be therefore confirmed in a RCT study adjusting for these limitations.

Future studies should examine the effects of a PNE program which consists of at least two education sessions and to combine PNE with another physical intervention. In addition, studies should focus on the prevention of persistent pain following cancer by introducing PNE before cancer treatment. Because of the small sample size, the influences of PNE were analyzed on a group level. Since people with different types of cancer might respond differently to PNE, future studies differentiating between cancer types and treatment might be of value. Once its use is supported by randomized controlled trials in more specific cancer survivor populations, PNE should be incorporated within the multidisciplinary treatment of cancer. PNE can be provided by rehabilitation nurses or other rehabilitation providers for preventive (pre-cancer treatment) and/or curative (post-cancer treatment) intent. To increase feasibility within inpatient hospital care, the one-on-one PNE sessions might be replaced by group sessions. In 
addition, the format of the PNE could be incorporated online, providing patients have the possibility to learn at own pace; thus decreasing the work load for the health care professional (Malfliet et al 2018).

\section{CONCLUSION}

Educating the cancer survivor about the neurophysiology of pain provides the cancer survivor with information about the various concepts about pain. Information about the persistence of pain, despite being told the cancer is gone, decreases the patients' rumination about painful sensations. In addition, when patients apply the PNE knowledge and develop new insights about pain in their daily life, their perceived inability to control pain (helplessness) improves. Although the current results need to be verified in a larger randomized, controlled trial, preliminary evidence shows that one session of PNE was associated with reductions in pain intensity and pain catastrophizing in cancer survivors with persistent pain. 


\section{Clinical relevance}

Implementing PNE before or after cancer treatment might reduce pain intensity and pain catastrophizing in cancer survivors.

\section{Acknowledgements}

This work was supported by a Chair funded by the Berekuyl Academy / European College for Decongestive Lymphatic Therapy, the Netherlands and awarded to the Vrije Universiteit Brussel, Belgium. 


\section{References}

Adam R, Bond C \& Murchie P 2015 Educational interventions for cancer pain. A systematic review of systematic reviews with nested narrative review of randomized controlled trials. Patient Education and Counseling 98: 269-282 doi:10.1016/j.pec.2014.11.003

Bennett MI, Bagnall AM \& José Closs S 2009 How effective are patient-based educational interventions in the management of cancer pain? Systematic review and meta-analysis. Pain 143: 192-199

doi:10.1016/j.pain.2009.01.016

Binkley JM, Harris SR, Levangie PK, Pearl M, Guglielmino J, Kraus V \& Rowden D 2012 Patient perspectives on breast cancer treatment side effects and the prospective surveillance model for physical rehabilitation for women with breast cancer. Cancer 118: $2207-2216$

doi:10.1002/cncr.27469

Bredal IS, Smeby NA \& Ottesen S 2014 Chronic Pain in Breast Cancer Survivors : Comparison of Psychosocial , Surgical , and Medical Characteristics Between Survivors With and Without Pain. Journal of Pain and Symptom Management 48(5):852-62 doi:10.1016/j.jpainsymman.2013.12.239

Clauw DJ 2015 Diagnosing and treating chronic musculoskeletal pain based on the underlying mechanism(s). Best Practice \& Research Clinical Rheumatology: 1-14 doi:10.1016/j.berh.2015.04.024

Cleeland CS, Allen JD, Roberts SA, Brell JM, Giralt SA, Khakoo AY, Kirch RA, Kwitkowski VE, Liao Z \& Skillings J 2012 Reducing the toxicity of cancer therapy: Recognizing needs, 
taking action. Nature Reviews Clinical Oncology 9: 471-478

doi:10.1038/nrclinonc.2012.99

De Groef A, De Vrieze T, Vos L, Van Kampen M, Geraerts I, Devoogdt N \& Meeus M 2018 Unraveling self-reported signs of central sensitization in breast cancer survivors with upper limb pain: Prevalence rate and contributing factors. Pain Physician 21: 27-256

Ferlay J, Colombet M, Soerjomataram I, Mathers C, Parkin DM, Piñeros M \& Bray F 2019 Estimating the global cancer incidence and mortality in 2018: GLOBOCAN sources and methods. International Journal of Cancer 144: 1941-1953 doi:10.1002/ijc.31937

Ferlay J, Shin HR, Bray F, Forman D, Mathers C \& Parkin DM 2010 Estimates of worldwide burden of cancer in 2008: GLOBOCAN 2008. International Journal of Cancer 127: $2893-2917$

doi:10.1002/ijc.25516

De Angelis R, Sant M, Coleman MP, Francisci S, Baili P, Pierannunzio D, Trama A, Visser O, Brenner H, Ardanaz E, Bielska-Lasota M, Engholm G, Nennecke A, Siesling S, Berrino F, Capocaccia R, \& EUROCARE-5 Working group 2014 Cancer survival in Europe 19992007 by country and age: Results of EUROCARE-5 - A population-based study. The Lancet Oncology 15: 23-34

doi:10.1016/S1470-2045(13)70546-1

Geneen LJ, Martin DJ, Adams N, Clarke C, Dunbar M, Jones D, McNamee Paul, Schofield P \& Smith BH 2015 Effects of education to facilitate knowledge about chronic pain for adults : a systematic review with meta-analysis. Systematic Reviews 4: 132 doi:10.1186/s13643-015-0120-5 
Glare PA, Davies PS, Finlay E, Gulati A, Lemanne D, Moryl N, Oeffinger KC, Paice JA, Stubblefield MD \& Syrjala KL 2014 Pain in cancer survivors. Journal of Clinical Oncology 32: $1739-1747$

doi:10.1200/JCO.2013.52.4629

Harrington CB, Hansen JA, Moskowitz M, Todd BL \& Feuerstein M 2010 It's Not over When it's Over: Long-Term Symptoms in Cancer Survivors-A Systematic Review. The International Journal of Psychiatry in Medicine 40: 163-181 doi:10.2190/PM.40.2.c

Hungr C, Sanchez-Varela V \& Bober SL 2017 Self-Image and Sexuality Issues among Young Women with Breast Cancer: Practical Recommendations. Rev Inves Clin 69: 114122

Jensen MP 2003 The validity and reliability of pain measures in adults with cancer. Journal of Pain 4: 2-21 doi:10.1054/jpai.2003.1

Kudel I, Edwards RR, Kozachik S, Block BM, Agarwal S, Heinberg LJ, Haythornthwaite J \& Raja SN 2007 Predictors and consequences of multiple persistent postmastectomy pains. J Pain Symptom Manage 34: 619-627 doi:10.1016/j.jpainsymman.2007.01.013

Lee I, Garland SN, Demichele A, Farrar JT, Im EO \& Mao JJ 2017 A cross-sectional survey of pain catastrophising and acupuncture use among breast cancer survivors. Acupuncture in Medicine 35: 38-43 doi:10.1136/acupmed-2016-011056 
Levy MH, Chwistek M \& Mehta RS 2008 Management of Chronic Pain in Cancer Survivors. The Cancer Journal 14: 401-409

doi:10.1097/PP0.0b013e31818f5aa7

Leysen L, Adriaenssens N, Nijs J, Pas R, Bilterys T, Vermeir S, Lahousse A \& Beckwée D 2018 Chronic pain in breast cancer survivors: nociceptive, neuropathic or central sensitization pain? Pain Practice: 0-3

doi:10.1111/papr.12732

Leysen L, Lahousse A, Nijs J, Adriaenssens N, Mairesse O, Ivakhnov S, Biltereys T, Van Looveren E, Pas R \& Beckwée D 2019 Prevalence of pain in breast cancer survivors: a systematic review and meta-analysis. Support Care Cancer 27: 4401-4433 doi: $10.1007 / \mathrm{s} 00520-019-04936-5$.

Louw A, Puentedura EJ, Zimney K \& Schmidt S 2016 Know Pain, Know Gain? A Perspective on Pain Neuroscience Education in Physical Therapy. J Orthop Sports Phys Ther 46: 131-134

doi:10.2519/jospt.2016.0602

Louw A, Zimney K, Puentedura EJ, Diener I 2016 The efficacy of pain neuroscience education on musculoskeletal pain : A systematic review of the literature. Physiotherapy Theory and Practice 32: 332-355

doi:10.1080/09593985.2016.1194646

Lovell MR, Luckett T, Boyle FM, Phillips J, Agar M \& Davidson PM 2014 Patient education, coaching, and self-management for cancer pain. J Clin Oncol 32: 1712-1720 doi:10.1200/JCO.2013.52.4850 
Malfliet A, Kregel J, Meeus M, Roussel N, Danneels L, Cagnie B, Dolphens M \& Nijs J 2018 Blended-Learning Pain Neuroscience Education for People With Chronic Spinal Pain: Randomized Controlled Multicenter Trial. Physical therapy 98: 357-368

Malfliet A, Leysen L, Pas R, Kuppens K, Nijs J, Van Wilgen P, Huysmans E, Goudman L \& Ickmans K 2017 Modern pain neuroscience in clinical practice: applied to post-cancer, paediatric and sports-related pain. Brazilian Journal of Physical Therapy 21: 225-232 doi:10.1016/j.bjpt.2017.05.009

McGuire DB 2004 Occurrence of Cancer Pain. Journal of the National Cancer Institute Monographs 51-56

doi:10.1093/jncimonographs/lgh015

Meeus M, Nijs J, Van Oosterwijck J, Van Alsenoy V \& Truijen S 2010 Pain physiology education improves pain beliefs in patients with chronic fatigue syndrome compared with pacing and self-management education: A double-blind randomized controlled trial. Archives of Physical Medicine and Rehabilitation 91: 1153-1159

doi:10.1016/j.apmr.2010.04.020

Merskey H \& Bogduk N 1994 IASP Task Force on Taxonomy Part III: Pain Terms, A Current List with Definitions and Notes on Usage. IASP Task Force on Taxonomy: 209214 doi:http://dx.doi.org/10.1016/0304-3959(86)90113-2

Nijs J, Wijma AJ, Leysen L, Pas R, Willaert W, Hoelen W, Ickmans K \& Wilgen CPV (2018). Explaining pain following cancer: a practical guide for clinicians. Brazilian Journal of Physical Therapy. 
doi:10.1016/j.bjpt.2018.12.003

Rosenthal JA 1996 Qualitative Descriptors of Strength of Association and Effect Size. Journal of Social Service Research 21: 37-59

doi:10.1300/J079v21n04_02

Rosenthal R 1994 Parametric measures of effect size. New York: Russell Sage Foundation.

Schreiber KL, Kehlet H, Belfer I \& Edwards RR 2014 Predicting, preventing and managing persistent pain after breast cancer surgery: the importance of psychosocial factors. Pain management 4: 445-459

doi:10.2217/pmt.14.33

Sun Y, Shigaki CL \& Armer JM 2017 Return to work among breast cancer survivors: A literature review. Support Care Cancer 25: 709-718

doi:10.1007/s00520-016-3446-1

Syrjala KL, Jensen MP, Elena Mendoza M, Yi JC, Fisher HM \& Keefe FJ 2014 Psychological and behavioral approaches to cancer pain management. Journal of Clinical Oncology 32: $1703-1711$

doi:10.1200/JCO.2013.54.4825

Treanor C \& Donnelly M 2014 A methodological review of the Short Form Health Survey 36 (SF-36) and its derivatives among breast cancer survivors. Quality of Life Research 24: $339-362$

doi:10.1007/s11136-014-0785-6 
Van Damme S, Crombez G, Bijttebier P, Goubert L \& Van Houdenhove B 2002 A confirmatory factor analysis of the Pain Catastrophizing Scale: Invariant factor structure across clinical and non-clinical populations. Pain 96: 319-324 doi:10.1016/S0304-3959(01)00463-8

Van Oosterwijck J, Nijs J, Meeus M, Truijen S, Craps J, Van den Keybus N \& Paul L 2011 Pain neurophysiology education improves cognitions, pain thresholds, and movement performance in people with chronic whiplash: A pilot study. The Journal of Rehabilitation Research and Development 48: 43-58 doi:10.1682/JRRD.2009.12.0206 
Table 1. Effect of PNE on pain intensity, pain catastrophizing and health related quality of life

\begin{tabular}{|c|c|c|c|c|c|c|}
\hline Outcome measures & $\begin{array}{l}\text { Pre-PNE }(\mathbf{n}=\mathbf{3 0}) \\
\text { Median }(I Q R)\end{array}$ & $\begin{array}{l}\text { Post-PNE }(\mathbf{n}=\mathbf{3 0}) \\
\text { Median (IQR) }\end{array}$ & $\begin{array}{c}\text { Median } \\
\text { difference }\end{array}$ & $p$ & $T$ & $r$ \\
\hline $\begin{array}{l}\text { VAS } \\
\text { PCS }\end{array}$ & $47(43-65)$ & $40(34-55)$ & -6 & 0.001 & 46 & -.44 \\
\hline Rumination & $7(4-10.25)$ & $5.5(2-9)$ & -1 & $0.002 *$ & 19.5 & -.39 \\
\hline Magnification & $4(0.75-6)$ & $3.5(1-5)$ & 0 & 0.095 & 48.5 & -.22 \\
\hline Helplessness & $8(4-13)$ & $6(1.75-10.25)$ & -1 & $<0.001 *$ & 30.5 & -.48 \\
\hline $\begin{array}{l}\text { Total } \\
\text { SF-36 }\end{array}$ & $16.5(10.75-28)$ & $16.5(5-25.25)$ & -3 & $<0.001 *$ & 36.0 & -.46 \\
\hline Physical Functioning & $55(46.25-81.25)$ & $60(33.75-81.25)$ & 0 & 0.813 & 36.0 & -.03 \\
\hline Social Functioning & $62.5(50-78.13)$ & $62.5(50-75)$ & 0 & 0.891 & 82.5 & -.02 \\
\hline Role Physical & $12.5(0-81.25)$ & $25(0-100)$ & 0 & 0.357 & 7.5 & -.12 \\
\hline Role Emotional & $66.7(33.33-100)$ & $66.7(33.33-100)$ & 0 & 0.603 & 11.0 & -.07 \\
\hline Emotional wellbeing & $72(63-84)$ & $74(63-84)$ & 0 & 0.372 & 38.5 & -.12 \\
\hline Energy \& Fatigue & $52.5(43.75-65)$ & $55(40-70)$ & 0 & 0.363 & 57.5 & -.12 \\
\hline Pain & $45(44.38-67.50)$ & $55(45-70)$ & 10 & $0.003 *$ & 25.0 & -.39 \\
\hline General Health & $35(33.75-55)$ & $40(33.75-57.5)$ & 0 & 0.145 & 34.5 & -.19 \\
\hline Health Changes & $50(25-75)$ & $50(25-75)$ & 0 & 0.748 & 9.0 & -.04 \\
\hline
\end{tabular}

Analysis was performed with the Wilcoxon signed- rank test.

Pre-PNE: Before the Pain Neuroscience Education session, Post-PNE: After the Pain Neuroscience Education session, IQR: Interquartile range, Median difference: median difference between the Pre-PNE measure and Post-PNE measure within the participants, $\boldsymbol{p}: \boldsymbol{p}$ - 
value, *: $\mathrm{p}<0.05, \boldsymbol{T}$ : T-value, $\boldsymbol{r}$ : effect size (correlation), VAS: Visual Analogue Scale, PCS: Pain Catastrophizing Scale, SF-36: Short Form 36 
Figure 1. Timetable study protocol

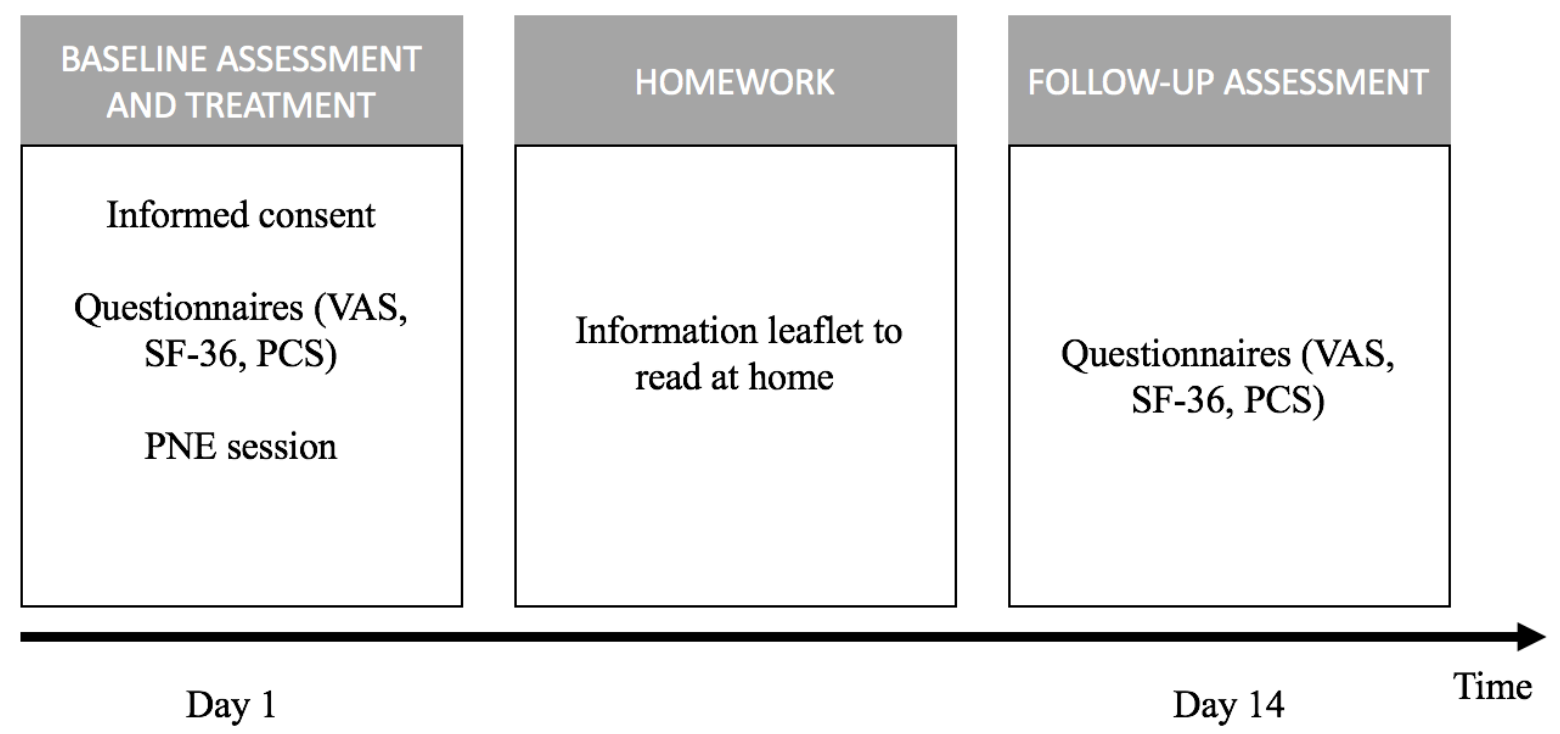

PNE: Pain Neuroscience Education; VAS: Visual Analogue Scale; PCS: Pain

Catastrophizing Scale; SF-36: Short Form 36 Health Status Survey 